\title{
Opacification of intraocular lens implant after uncomplicated cataract surgery: A case series
}

\author{
Ching-Lin Wong, ${ }^{1,2}$ K.S. Sendhil, ${ }^{1,3}$ T.V.N. Karunakar, ${ }^{1}$ \\ Ming-Yueh Lee ${ }^{1,4}$ \\ 'Department of Ophthalmology, Kuala Lumpur Hospital, Kuala Lumpur, Malaysia; \\ ${ }^{2}$ Department of Ophthalmology, Penang Hospital, Penang, Malaysia; ${ }^{3}$ Department \\ of Ophthalmology, Sir Charles Gairdner Hospital, Australia; ${ }^{4}$ Department of \\ Ophthalmology, Tuanku Ja'afar Hospital, Negeri Sembilan, Malaysia
}

\begin{abstract}
Opacification of intraocular lens implant after successful cataract surgery is an important issue with the introduction of new intraocular lens. The complexity of the problem is not merely associated with visual impairment, but also lies in the difficulty in diagnosis and management of the case. We report 3 cases of late postoperative opacification of IOL implants warranted IOL exchange.
\end{abstract}

Key words: Opacification, intraocular lens

\section{Introduction}

Transparency in an implanted intraocular lens (IOL) is essential for the maintenance of high-quality vision after cataract extraction. Although advancement in lens manufacturing and lens design have significantly improved lens clarity and ultimate visual outcomes of cataract surgery, loss of IOL transparency over time is still a potential problem that could end up with IOL explantation. Postoperative opacification of IOL has been reported in all varieties of lens including silicone, hydrophobic acrylic, hydrophilic acrylic, hydrogel and even in PMMA lens.' We report three cases of late postoperative opacification of IOL implants warranted IOL exchange.

\section{Case 1}

A 55-year-old Chinese female with diabetes and hypertension had undergone uneventful left phacoemulsification cataract surgery with posterior chamber Acriflex 50CSE Hydrophilic Acrylic IOL (Acrimed) in the left eye in April 2006 with best-corrected visual acuity (BCVA) of $6 / 24$ post-operatively. She came back two years later with profound drop in vision to perception to light. Examination revealed homogenous white opacification of the optical component of IOL (Figs. 1 and 2). She underwent an eventful IOL explantation with anterior vitrectomy and anterior chamber IOL implantation (PMMA). However, her post-operative BCVA was not much improved, $6 / 60$ due to her existing diabetic maculopathy.

Correspondence: Correspondence: Dr Ming Yueh Lee, Department of Ophthalmology, Kuala Lumpur Hospital, 50586 Jalan Pahang, Kuala Lumpur, Malaysia.

E-mail: leemingyueh@yahoo.com 


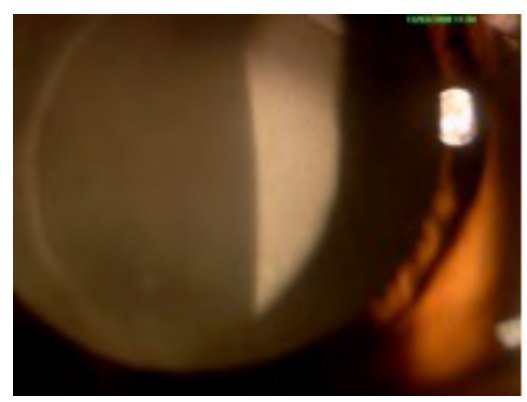

Fig. 1. Slit-lamp photo showing opacified Acriflex 50CSE Hydrophilic Acrylic IOL (Acrimed).

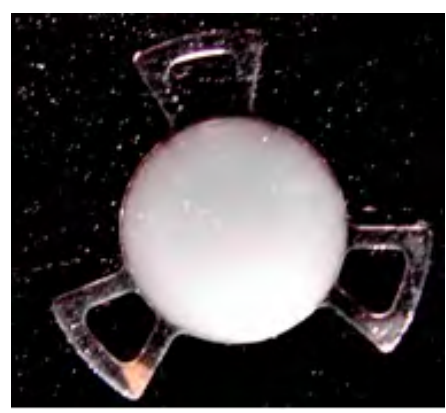

Fig. 2. Explanted single piece Acriflex 50CSE Hydrophilic Acrylic IOL (Acrimed).

\section{Case 2}

A 60-year-old Indian female, also a known case of diabetes, had right phacoemulsification cataract surgery with in-the-bag posterior chamber Hydroview H60M IOL (Bausch and Lomb Surgical; Rochester, NY, USA) implantation in August 1999. Her BCVA post-operatively was $6 / 6$. She presented seven years later, with vision of counting finger! A diagnosis of non-resolving vitreous hemorrhage was made and she was referred to the Vitreo-retina team for vitrectomy. Further examination pointed to opacification of the implanted IOL (Fig. 3). The vitreous was confirmed clear on B scan. She underwent successful IOL explantation with secondary IOL implantation (hydrophobic-acrylic) in the sulcus (Fig. 4). Her postoperative visual acuity was best corrected to $6 / 6$.

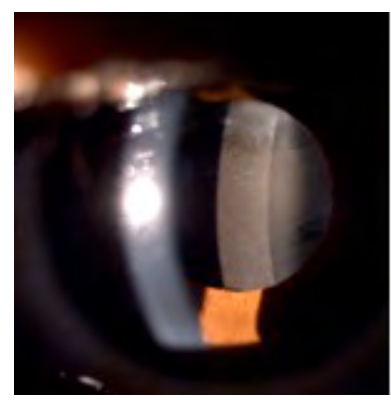

Fig. 3. Slit-lamp photos showing opacified Hydroview H6OM IOL.

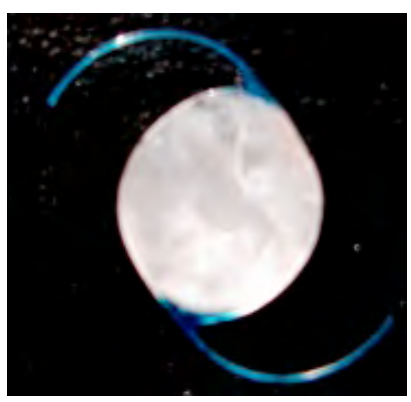

Fig. 4. Explanted single-piece Hydroview H60M IOL.

\section{Case 3}

In October 2007, a 72 year-old Chinese female diabetic, with hypertension and ischemic heart disease and suffering from open-angle glaucoma was referred to the Vitreo-Retina Team for further management of vitreous hemorrhage in the left eye. She had a history of uneventful phacoemulsification cataract surgery 
with posterior chamber Acriflex 50CSE Hydrophilic Acrylic IOL (Acrimed) implantation one year before. Vision was hand-movement on presentation. Examination revealed an opaque IOL in the bag which warranted explantation. The release of the IOL was difficult due to fibrosed capsule with capture of IOL haptic. The IOL was maneuvered into the anterior chamber and delivered through a limbal wound. (The explanted IOL had a similar appearance as that in Case 1.) She was left aphakic in view of zonular dialysis. A secondary implantation of posterior chamber IOL in the capsular bag was performed in August 2008. Postoperatively, her BCVA was $6 / 12$.

\section{Discussion}

Opacification of the intraocular lens (IOL) is an often overlooked cause of visual impairment following uneventful cataract surgery. It is often misdiagnosed as posterior capsule opacification or vitreous opacification and may unduly be subjected to Yag capsulotomy or vitreo-retinal surgery.

General factors contributing to postoperative IOL opacification could be IOL biomaterial and manufacturing, IOL packaging, surgical technique or patient's factors. IOL biomaterial like acrylic, hydrophilic, hydrogel and silicone had shown to have a higher incidence of IOL opacification post-cataract surgery among all the available IOL. ${ }^{1}$ A packaging gasket containing silicone had been implicated in cases of IOL opacification. ${ }^{2}$ Inadvertent damage of the optical surface by surgical instruments could promote calcium deposit postoperatively. The patient's pre-existing conditions, particularly her diabetes and glaucoma, have been postulated as a precipitating risk factor. ${ }^{3}$

Opacification of Acriflex 50CSE Hydrophilic Acrylic IOL (Acrimed) with resultant total recall of 239 patients, was reported by Andrew Lim and colleagues in Selayang Hospital, Malaysia. It involved $5.4 \%$ cases implanted with this hydrophilic acrylic lens. Analysis with scanning electron microscopy and energy dyspersive X-Ray spectroscopy of these cases revealed surface calcium and phosphorus deposits and intralenticular traces of calcium in the opacified IOL. ${ }^{4} \mathrm{It}$ is believed that the hydroxyhexyl methacylate present in Acriflex 50CSE Hydrophilic Acrylic IOL (Acrimed) has calcium affinity properties which were responsible for the lens calcification. The other type of hydrophilic acrylic IOL which had been reported with significant postoperative IOL opacification requiring $\mathrm{IOL}$ exchange was SC60B-0UV. ${ }^{5}$

Hydroview $\mathrm{H} 60 \mathrm{M} \mathrm{IOL}$ with significant lens opacification in $4.2 \%$ of post-cataract surgery cases warranting $\mathrm{IOL}$ exchange had been reported by Balasubramaniam et al. ${ }^{3}$ Dorey et al. pointed out that opacified Hydroview H6OM IOL was due to silicon acting as a nidus for calcium deposition; which was presumably derived from the Surefold packaging system. ${ }^{2}$ Chemistry analysis on the surface deposits of the opacified Hydroview H60M IOL identified a mixture of calcium, fatty acid, salt and silicone. ${ }^{1,6}$ Opacification of H60M IOL reported most commonly occurred between 12 and 25 months postoperatively. Our case was exceptional because of its late presentation. 
Opacification of Silicon IOL was the most commonly reported cause of IOL opacification in patients who underwent vitreo-retina surgery with silicon oil tamponade. Silicon oil in a vitrectomized eye can also adhere to any hydrophobic, hydrophilic and even PMMA lens resulting in IOL opacification. Other reported cases of IOL opacification were PMMA IOL secondary to late biodegradation of PMMA and Acrysof ${ }^{\mathrm{TM}}$ 'glistening' with microvacuole formation in the lens.'

Patient factors like metabolic imbalance, breakdown of the blood-aqueous barrier and high level of calcium and phosphorus in the aqueous and serum had been implicated as causes of lens opacification in the diabetics; ${ }^{1,2}$ which could be the case in the three patients in this series.

Opacified IOL is often misdiagnosed due to poor fundus view. Haymore et al. ${ }^{7}$ reported eight cases of misdiagnosis of hydrophilic acrylic lens optic opacification. Four were misdiagnosed as having posterior capsular opacification and underwent $\mathrm{Nd}$ :YAG laser capsulotomy and the other four were misdiagnosed as having vitreous opacities and were subjected to vitrectomies. Failure to recognize the process of $\mathrm{IOL}$ opacification may lead to unwarranted surgical procedures.

Explantation and exchange of $\mathrm{IOL}$ is the only solution to $\mathrm{OLL}$ opacification. $\mathrm{Nd}$ :Yag laser treatment is not effective in clearing the opacification. Instead it could jeopardize implantation of a new IOL into the capsular bag. Nevertheless, explantation could be technically difficult and challenging. Posterior capsule rupture, zonular dehiscence, iridodialysis and vitreous loss are common complications seen in $10-48 \%$ of the $\mathrm{IOL}$ exchanges. ${ }^{5}$

Meticulous surgical technique is necessary in IOL removal. The anterior capsule should be carefully teased off the IOL. Careful visco-dissection and radial cuts on the anterior capsule can facilitate the procedure. It is important to caution that attempts to rotate the $\mathrm{IOL}$ out of the bag risk zonular dehiscence and rupture of the posterior capsule. Once the IOL is delivered into the anterior chamber, it can be delivered in one piece through a slightly bigger limbal incision the way in which it was done in our patients. Alternatively, it can be cut into two halves and retrieved through a 4-mm limbal incision. Very often the haptic was retained in the fibrosed capsule making complete explantation of IOL impossible. ${ }^{5}$ In case of ruptured posterior capsule, the secondary IOL could be sulcus-fixated if in-the-bag implantation is not feasible. In the event of inadequate capsular support, anterior chamber IOL or scleral fixated IOL should be the alternatives. Besides the difficult surgical procedure in IOL explantation, the visual outcome after successful IOL exchange was not promising. Many patients developed post-operative cystoid macular edema after IOL exchange. ${ }^{5}$ Therefore, prescription of topical NSAID, a form of 'off-label' treatment which is widely used is advisable after a complicated surgical procedure. Control of postoperative inflammation with steroids is equally important to minimize the risk of post-operative cystoid macular edema. Subtenon, infra-orbital or intravitreal triamcinolone could be given for severe inflammation. Medical therapy with systemic acetazolamide is one of the options besides intravitreal anti-VEGF injection. Surgical intervention is required for macular oedema secondary to vitreo-macular traction. ${ }^{8}$ 
Although IOL opacification postoperatively is a rare entity, careful selection of IOL should not be overlooked. It is particularly important for patients with present or potential vitreo-retinal disease such as diabetics and high myopic patients who may need silicone oil as tamponade to avoid having silicone IOL.

We recommend that new IOL should be rigorously tested for longer period by researchers before large-scale usage. Informed consent of cataract surgery should also include the possibility of postoperative IOL opacification. A proper reporting system is useful to highlight the issue and to alert the other clinicians of the potential hazard of postoperative opacification of particular IOL. Adverseevent reporting systems have been implemented in some countries to allow early reporting of problems with IOL. ${ }^{4}$ Further investigations should be carried out to identify the cause of the IOL opacification and a notice or recommendation should be issued to withhold the use of the particular IOL or to alert caution in using it. This is to prevent repetition of the same problems of opacification of the particular IOL; especially in the under-privileged area where expertise is less available to handle this type of visual loss.

\section{Conclusion}

Careful testing and selection of new IOL for cataract surgery is important to minimize the incidence of $\mathrm{OOL}$ opacification postoperatively for it is a potential cause of significant visual impairment after cataract surgery. Furthermore, IOL exchange is a challenging procedure and may have an unpredictable surgical outcome.

\section{References}

1. Trivedi RH, Weiner L, Apple DJ, et al. Post cataract IOL surgey opacification. Eye 2002;16:217-241.

2. Dorey MW, Brownstein S, Hill VE, et al., Proposed pathogenesis for the delayed postoperative opacification of the hydroview hydrogel intraocular lens. Am J Ophthalmol 2003;135(5):591-598.

3. Balasubramaniam C, Goodfellow J, Kirkpatrick N. Opacification of the Hydroview H60M intraocular lens: Total patient recall. J Cataract Refract Surg 2006;32(6):944-948.

4. Andrew KE Lim, PP Goh, Azura R, Mariam I. Opacification of Aceiflex 50CSE hydrophilic acrylic intraocular lenses. J Cataract Refract Surg 2011;37:655-659.

5. Gashau AG, Anand A, Chawdhary S. Hydrophilic acrylic intraocular lens exchange: Five-year experience. J Cataract Refract Surg 2006;32(8):1340-1344.

6. Werner L, Apple DJ, Escobar-Gomez M, et al. Postoperative deposition of calcium on the surfaces of a hydrogel IOL. Ophthalmology 2000;107:2179-2185.

7. Haymore J, Zaidman G, Werner L, et al. Misdiagnosis of Hydrophilic Acrylic Intraocular Lens Optic Opacification: Report of 8 Cases with the MemoryLens. Ophthalmology 2007;114(9):1689-1695.

8. Zaidman G, Werner L, Arthur F, Ahmed I, Everett Al. Cystoid Macular Edema. In: Yanoff M, Duker JS, Ophthalmology: Retina and Vitreous. ${ }^{\text {nd }}$ ed. Philadelphia: Mosby 2004; pp. 956-962. 